\title{
Hormone Treatments during Maize Vegetative and Reproductive Development
}

María Jazmín Abraham-Juárez ${ }^{1, \$, *}$ and Harry Klein ${ }^{1}$

\author{
${ }^{1}$ Department of Biology, University of Massachusetts Amherst, Amherst MA, USA; ${ }^{\$}$ Current address: \\ CONACYT. División de Biología Molecular, Instituto Potosino de Investigación Científica y Tecnológica \\ (IPICYT), San Luis Potosí, SLP, Mexico \\ *For correspondence: maria.abraham@ipicyt.edu.mx
}

\begin{abstract}
[Abstract] Plant hormones are a group of organic natural compounds that can influence physiological processes when present at low concentrations. Plant biologists frequently use exogenous hormone application to modify plant physiology or to rescue mutants with defects in hormone pathways. There are a number of methods for these treatments, depending on the plant species, organs and tissues used for application, release path, among others. Here, we describe a simple method used for hormone treatment in maize plants. At the vegetative stage, we show the application of Gibberellic acid $\left(\mathrm{GA}_{3}\right)$ to wild type and dwarf1 (d1) mutants result in cell elongation (Evans et al., 1995). DWARF1 Encodes a Gibberellin 3-Oxidase enzyme (Chen et al., 2014), so the dwarf phenotype is rapidly recovered by $\mathrm{GA}_{3}$ application. In addition, wild type plants show rapid stem elongation in response to $\mathrm{GA}_{3}$ application. At the reproductive stage, we use our method to apply Jasmonic acid (JA) to a tasselseed2 (ts2) mutant tassel. TS2 suppresses carpels in the tassel and in lower florets in the ear and ts2 mutants can be rescued by exogenous JA application (DeLong et al., 1993). In addition to detailing the procedure for hormone treatments, we discuss potential issues and other aspects worth considering when performing these methods.
\end{abstract}

Keywords: Maize, Hormones, Vegetative, Reproductive, Development

\section{Materials and Reagents}

1. Sterile 15 and $50 \mathrm{ml}$ tubes

2. Sterile pipette tips $(1,000,200$ and $10 \mu l)$

3. Ruler (for determination of plant growth)

4. Pots (3.5-inch square pots and 3-gallon pots) (Hummert International, T.O. Plastics)

5. Supersoil ${ }^{\circledR}$ Potting Soil (for growing plants)

6. Maize seeds of the desired genotypes
a. Wild type: B73
b. Mutants: dwarf1 (d1), tasselssed2 (ts2)

7. Gibberellic acid $\left(\mathrm{GA}_{3}\right)$ (Sigma-Aldrich, catalog number: 36575 )

8. Jasmonic acid (JA) (Sigma-Aldrich, catalog number: J2500)

9. Tween 20 (Sigma-Aldrich, catalog number: P9416)

10. Absolute ethanol 
11. Epibrassinolide (Sigma-Aldrich, catalog number: E1641)

12. $\mathrm{GA}_{3}$ treatment (see Recipes)

13. $G A_{3}$ mock (see Recipes)

14. JA treatment (see Recipes)

15. JA mock (see Recipes)

16. BR treatment (see Recipes)

17. BR mock (see Recipes)

18. IAA treatment (see Recipes)

19. IAA mock (see Recipes)

\section{Equipment}

1. Micropipettes $(1,000,200$ and $10 \mu \mathrm{l})$

2. Camera

\section{Procedure}

A. Hormone treatments during maize vegetative development

1. Sow maize kernels under $2 \mathrm{~cm}$ potting soil in 3.5-inch pots and grow for a week (see Note 1). Greenhouse conditions are $25{ }^{\circ} \mathrm{C}, 50 \%$ relative humidity, with $14 \mathrm{~h}$ of light at $100 \mathrm{mmol} \cdot \mathrm{m}^{-2} \cdot \mathrm{s}^{-1}$. Ideally, treatment should begin just before the developmental stage in question. It is recommended that 15 kernels at least be sowed for each data point to ensure you will get at least 10 plants for each genotype (e.g., wild type and mutant) per treatment (mock and hormone) for analysis.

2. When three leaves are visible in the plants (V2 stage) (Figure 1), begin the treatment. Always apply mock and hormone treatment to each genotype to have adequate controls. Applications should be done at the same time of the day during all the treatment period to account for differences in endogenous metabolite levels.

3. For hormone application, use $200 \mu$ l of stock solution. Apply the total volume to the apical whorl of seedlings (Figure 1). 


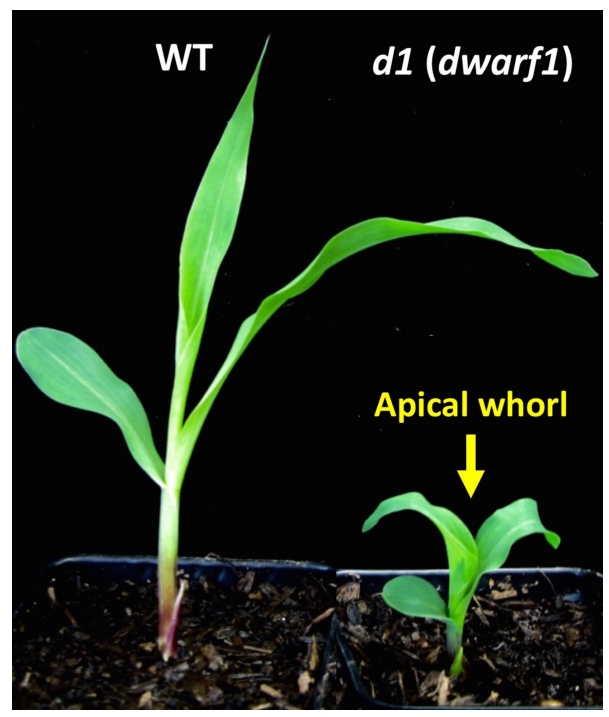

Figure 1. Wild type (B73) and dwarf1 mutant used for hormone treatment. Plants are at V2 maize developmental stage.

4. Repeat applications every $48 \mathrm{~h}$, during 2 weeks or until treated plants show significant changes in phenotype compared to the negative control (Figure 2).

5. Record the effect of the treatment by taking pictures over the time. Make measurements every other day to have representative variations (Figure 2). Stem length is measured from the base of the stem to the last emerged ligule.
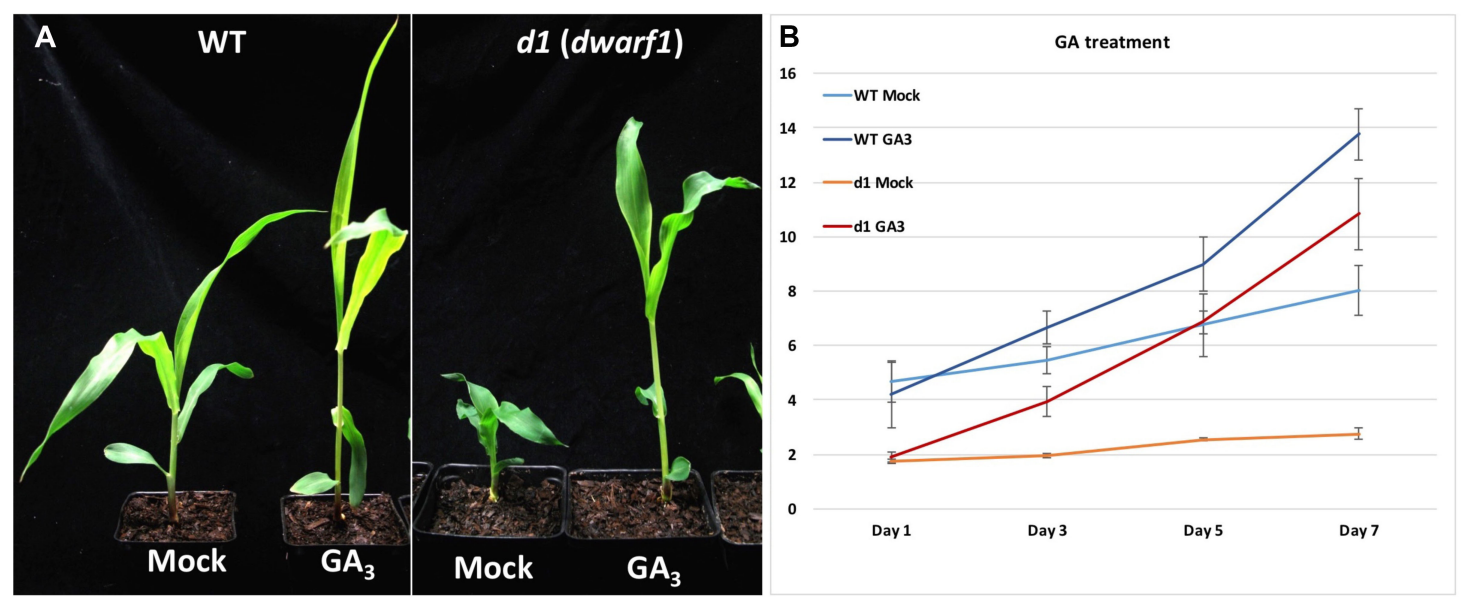

Figure 2. Plant height $(\mathrm{cm})$ after $\mathrm{GA}_{3}$ treatment in wild type and $\mathbf{d} 1$ mutant. A. Pictures were taken seven days after the first application. B. Height measurements were taken every other day to graph the average. Ten plants for each genotype and treatment were used.

B. Hormone treatments during maize reproductive development

1. Sow maize kernels under $2 \mathrm{~cm}$ potting soil in 3-gallon pots and grow for 4-5 weeks (see Note 1). 
2. At 6 weeks, sample one of the largest plants to determine the tassel stage of development. Tassel staging depends on genotype and growing conditions. Ideally, treatment should begin just before the developmental stage in question. To analyze carpel suppression phenotypes, begin treatment at 0.7-1.0 cm length tassel. Tassels are measured from the base on the main spike to the apex (Figure 3).

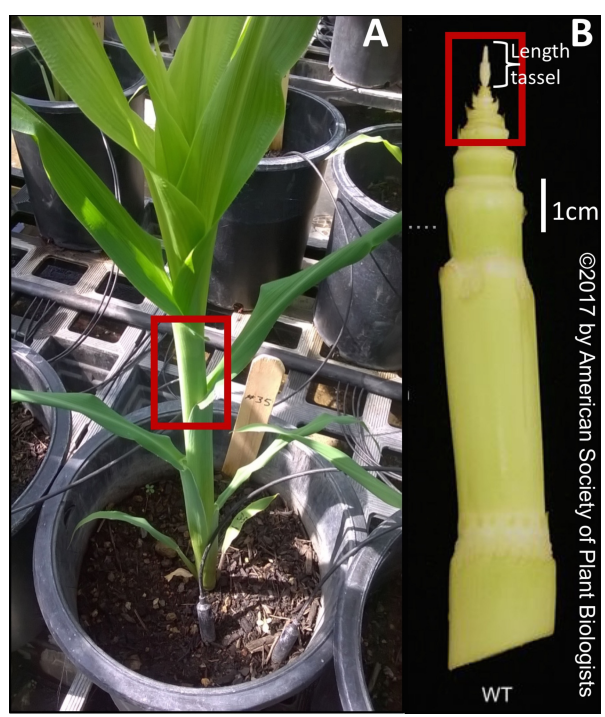

Figure 3. Tassel measurement. A. A whole plant at 7 weeks old. The red rectangle shows the apex position. B. Stem after removing leaves showing the tassel in the tip (Picture from Tsuda et al., 2017). A 0.7-1.0 cm tassel is shown.

3. Apply $1 \mathrm{ml}$ of stock solution (treatment or mock) to the apical whorl of plants (Figure 4). Similar to vegetative treatments, applications should be done at the same time of the day to account for differences in endogenous metabolite levels

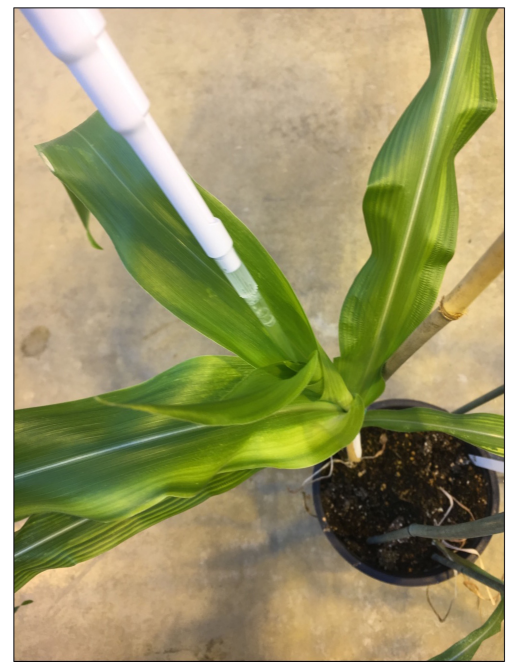

Figure 4. Application of JA to a six-week-old ts2 mutant 
4. Repeat applications every $48 \mathrm{~h}$ until tassel emergence (16 to 20 days) or until treated plants show significant changes in phenotype compared with the negative control (Figure 5).

5. Record the effect of the treatment by taking pictures of the inflorescence development (Figure 5).

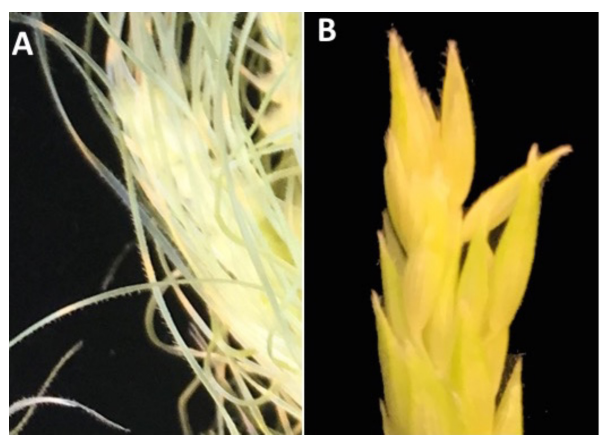

Figure 5. Maize tassels of ts2 mutant after mock (A) and JA (B) treatment. A. Tassel florets showing developed carpels after the mock treatment. B. Tassel florets showing complete suppression of carpels after the JA treatment.

\section{Data analysis}

Qualitative analysis can be done by taking pictures over the time to record phenotype changes. Quantitative analysis is carried out by making graphs showing the height of the plants or other quantitative traits in at least three independent replicates. Statistical analysis can be done using the average and standard deviation of measurements for each point.

\section{Notes}

For details about planting and growing maize, refer to Hake and Lunde (2018).

\section{$\underline{\text { Recipes }}$}

The following solutions for different hormone treatments in maize have been used (Yan et al., 2012; Rosa et al., 2017).

Notes:

a. Store the hormone stock solutions at $4{ }^{\circ} \mathrm{C}$ for a week and protect from light.

b. Prepare the following solutions with $\mathrm{dd}_{2} \mathrm{O}$ water.

1. $\mathrm{GA}_{3}$ treatment $100 \mu \mathrm{M} \mathrm{GA} 3$ $0.1 \%$ Tween-20 in water

2. $\mathrm{GA}_{3}$ mock

$0.1 \%$ Tween-20 in water 
3. JA treatment

$1 \mathrm{mM} \mathrm{JA}$ (from $200 \mathrm{mM}$ stock in 100\% Ethanol) in 0.1\% Tween-20 in water

4. JA mock

$0.005 \%$ ethanol

$0.1 \%$ Tween-20 in water

5. BR treatment

$10 \mu \mathrm{M}$ Epibrassinolide

$0.1 \%$ Tween-20 in water

6. BR mock

$0.1 \%$ Tween-20 in water

7. IAA treatment

$100 \mu \mathrm{M}$ IAA

$0.1 \%$ Tween-20 in water

8. IAA mock

$0.1 \%$ Tween-20 in water

\section{Acknowledgments}

MJAJ was supported by UC-MEXUS CONACYT and by NSF IOS-1238202 at the Dr. Sarah Hake Lab and by NSF IOS-1652380 at the Dr. Madelaine Bartlett Lab, where this protocol was developed. Authors would like to thank Dr. Sarah Hake and Dr. Madelaine Bartlett for their continuing scientific support. Authors declare no conflict of interest regarding the implementation of this protocol.

\section{References}

1. Chen, Y., Hou, M., Liu, L., Wu, S., Shen, Y., Ishiyama, K., Kobayashi, M., McCarty, D. R. and Tan, B. C. (2014). The maize DWARF1 encodes a gibberellin 3-oxidase and is dual localized to the nucleus and cytosol. Plant Physiol 166(4): 2028-2039.

2. DeLong, A., Calderon-Urrea, A. and Dellaporta, S. (1993). Sex determination gene TASSELSEED2 of maize encodes a short-chain alcohol dehydrogenase required for stagespecific floral organ abortion. Cell 74: 757-768.

3. Evans, M. M. and Poethig, R. S. (1995). Gibberellins promote vegetative phase change and reproductive maturity in maize. Plant Physiol 108(2): 475-487.

4. Hake, S. and Lunde, C. (2018). Growing and pollinating maize. Bio-101: e2832.

5. Rosa, M., Abraham-Juárez, M. J., Lewis, M. W., Fonseca, J. P., Tian, W., Ramirez, V., Luan, S., Pauly, M. and Hake, S. (2017). The maize MID-COMPLEMENTING ACTIVITY homolog CELL NUMBER REGULATOR13/NARROW ODD DWARF coordinates organ growth and tissue patterning. Plant Cell 29(3): 474-490. 
6. Tsuda, K., Abraham-Juarez, M. J., Maeno, A., Dong, Z., Aromdee, D., Meeley, R., Shiroishi, T., Nonomura, K. I. and Hake, S. (2017). KNOTTED1 cofactors, BLH12 and BLH14, regulate internode patterning and vein anastomosis in maize. Plant Cell 29(5): 1105-1118.

7. Yan, Y., Christensen, S., Isakeit, T., Engelberth, J., Meeley, R., Hayward, A., Emery, R. J. and Kolomiets, M. V. (2012). Disruption of OPR7 and OPR8 reveals the versatile functions of jasmonic acid in maize development and defense. Plant Cell 24(4): 1420-1436. 